\title{
Lesiones por disparo de escopeta. Necesidad de un punto de vista especial
}

\author{
Shotgun injuries. Need for a special point of view
}

Luis M. García-Núñez ${ }^{1,2 *}$, Gabriela A. Guillén-Hernández ${ }^{1,2}$, Edgar F. Hernández-García ${ }^{1,2}$, Óscar E. Pérez-Morales ${ }^{1,2}$, Jesús R. Ángeles-Varela ${ }^{1,2}$, Luis M. García-Espino3, Luis F. Moreno-Delgado ${ }^{1,2}$ y Héctor F. Noyola-Villalobos ${ }^{1,2}$

${ }^{1}$ Departamento de Cirugía General, Hospital Central Militar, Secretaría de la Defensa Nacional; '2Escuela Militar de Graduados de Sanidad, Universidad del Ejército y Fuerza Aérea, Secretaría de la Defensa Nacional; ${ }^{3}$ St. Luke Escuela de Medicina, Alliant International University. Ciudad de México, México

\section{Resumen}

Antecedentes: Las lesiones por disparo de escopeta (LE) son infrecuentes, pero debido a su balística especial se relacionan con un pronóstico adverso. Objetivo: Analizar variables operativas y administrativas de los pacientes con LE, de interés para la institución. Método: Se estudiaron retrospectivamente variables demográficas y asistenciales de pacientes con LE admitidos al Hospital Central Militar (Ciudad de México) entre julio de 2006 y agosto de 2019. Los métodos estadísticos usados fueron medidas de dispersión y frecuencia relativa de ocurrencia. Resultados: En 158 meses se admitieron 21 pacientes con LE. La edad media fue de $36.9 \pm 13.6$ años (rango: 14-61) y predominó el sexo masculino ( $n=20 ; 95 \%)$. La LE tipo II fue la más frecuente (62\%; 13/21). La estancia hospitalaria media fue de $37.1 \pm 28.7$ días (rango: 3-109) y en cuidados intensivos fue de $20.3 \pm 22.5$ días (rango: 3-99). La tasa global de morbilidad fue del 82\% (17/21) y las complicaciones más frecuentes fueron infecciosas. La tasa gobal de mortalidad fue del 24\% (5/21). Conclusiones: Las LE son causa de estancia hospitalaria prolongada, alta tasa de morbilidad y un enorme consumo de recursos humanos y materiales, por lo que las estrategias de prevención primaria son convenientes para los sistemas de salud comprometidos con el cuidado de estos casos.

Palabras clave: Lesión. Escopeta. Balística.

\section{Abstract}

Background: Shotgun injuries (SGI) are infrequent, but due its special ballistics, are related to adverse outcomes. Objective: To analyze operative and administrative variables, critical for the institution. Method: Demographics and health-care related variables from SGI patients admitted to Military Central Hospital (Mexico City) between July 2006 and August 2019, were retrospectively studied. Statistics methods used were dispersion measures and relative occurrence frequency. Results: Over a 15 months-span, 21 SGI patients were admitted. Mean age was $36.9 \pm 13.6$ years (range: 14-61) and male gender was dominant ( $n=20$; 95\%). Type II SGI were the most common injuries (62\%; 13/21). Mean hospital length of stay was $37.1 \pm 28.7$ days (range: $3-109$ ) while stay at ICU was $20.3 \pm 22.5$ days (range: $3-99)$. Global rate of morbidity was $82 \%$ (17/21)

\footnotetext{
Correspondencia:

*Luis M. García-Núñez

Enrique Sada Moguerza, 17

Centro Quirúrgico Satélite

Circuito Centro Comercial, Ciudad Satélite

Fecha de recepción: 25-09-2019

C.P. 53100, Naucalpan de Juárez, Edo. de México, México Fecha de aceptación: 08-01-2020

E-mail: Imgarcian@hotmail.com

DOI: $10.24875 / C I R U .20001600$

Cir Cir. 2020;88(4):500-507

Contents available at PubMed www.cirugiaycirujanos.com 0009-7411/@ 2020 Academia Mexicana de Cirugía. Publicado por Permanyer. Este es un artículo open access bajo la licencia CC BY-NC-ND (http://creativecommons.org/licenses/by-nc-nd/4.0/).
} 
and most frequent complications were infective. Global mortality rate was 24\% (5/21). Conclusions: Once SGI cause prolonged length of stay, high rate of morbidity and of enormous consuming of material and human resources, primary prevention strategies are convenient for health-care systems compromised to the care of these cases.

Key words: Injury. Shotgun. Ballistics.

\section{Introducción}

Las lesiones por disparo de escopeta (LE) son un importante problema de salud pública, sobre todo en países en emergencia'. Se calcula que el grupo más afectado son los varones de entre 20 y 29 años, y aunque representan solo un $0.5-2 \%$ del total de las heridas por proyectil de arma de fuego causan una significativa tasa de morbilidad relacionada con la enfermedad, consumen una gran cantidad de recursos humanos y materiales para su abordaje, y se relacionan con una tasa de mortalidad que no puede ser desestimada ${ }^{2-4}$.

Ya que la balística de efectos de las LE supone la presencia de traumatismos múltiples por postas individuales que varían en peso y tamaño, con diferente potencial de penetración, que se dispersan o concentran en un área de tamaño variable y que transmiten una cantidad de energía dependiente del tipo de arma y de la distancia de disparo, es esencial para el cirujano comprender que la anatomía de estas lesiones no es similar ni puede compararse con las producidas por los proyectiles de las armas de fuego convencionales, con independencia de la velocidad del misil. Esto debe llevar a la consciencia de que la estrategia de manejo de las LE permanece sin claridad, lo cual limita la capacidad de estudio del mecanismo de lesión, y el operador debe implementar la conducta diagnóstica y terapéutica más pertinente para optimizar los resultados concernientes a los índices de sobrevida y funcionalidad ${ }^{5}$.

Es interés primario del Grupo de Trauma del Hospital Central Militar analizar el panorama institucional, tanto epidemiológico como administrativo, que rodea al abordaje de estos pacientes, para evaluar los resultados obtenidos con su manejo y definir áreas de oportunidad, a fin de proponer e implementar estrategias de mejora en los protocolos específicos de manejo.

\section{Método}

La presente serie es un estudio retrospectivo (158 meses, de julio de 2006 a agosto de 2019) de aspectos epidemiológicos y administrativos de interés para la institución, en el que se analizan las variables obtenidas de los expedientes clínicos de los pacientes con LE admitidos al Hospital Central Militar de la Ciudad de México, que es un centro de trauma militar y urbano, y hospital de entrenamiento en cirugía de trauma de los cirujanos del instituto armado. Se estudiaron variables demográficas (sexo y edad), variables de arribo (presión arterial sistólica y diastólica, frecuencia cardiaca y respiratoria, puntuación de Glasgow y puntuación RTS [Revised Trauma Score] y puntuación ISS [Injury Severity Score]), intervenciones diagnósticas y terapéuticas al arribo, intervenciones quirúrgicas y adyuvantes realizadas, lesiones a órganos específicos y variables pronósticas (estancia hospitalaria, estancia en la unidad de cuidados intensivos, duración de la asistencia ventilatoria mecánica, tasa de morbilidad específica y global, tasa de mortalidad y causa y sitio de la muerte). Todas las variables se sujetaron a un análisis estadístico descriptivo con medidas de dispersión (media \pm desviación estándar) y frecuencia relativa de ocurrencia, usando el programa Microsoft ${ }^{\circledR}$ Excel $^{\circledR}$ para Office $365 \mathrm{MSO}$ (ProPlus, versión 1908). Cuando fue aplicable, debido al tamaño de la muestra, se empleó la prueba t de Student para realizar la comparación estadística.

\section{Resultados}

Durante un periodo de 158 meses (julio de 2006 a agosto de 2019) se admitieron al Hospital Central Militar 21 pacientes con LE (0.1 pacientes/mes), de los cuales 20 fueron del sexo masculino $(95.2 \%)$ y $1(4.8 \%)$ del sexo femenino, con una edad media de $36.9 \pm 13.6$ años (rango: 14-61). Los pacientes arribaron con presión arterial sistólica de $115.6 \pm 20.7 \mathrm{mmHg}$ (rango: 87-175) y diastólica de $76.1 \pm 12.7 \mathrm{mmHg}$ (rango: 54-104), frecuencia respiratoria de $23.1 \pm 6.1 \mathrm{resp} / \mathrm{min}$ (rango: 17-40), temperatura de $36.3 \pm 0.6^{\circ} \mathrm{C}$ (rango: 34.9-37.2), puntuación de Glasgow $14.7 \pm 0.6$ (rango: 13-15), puntuación RTS $6.91 \pm 1.11$ (rango: 4.79-7.84) y puntuación ISS $44.5 \pm 13.6$ (rango: 16-69), lo cual traduce la inclusión de una población de estudio estable fisiológicamente, pero con lesiones de alto 


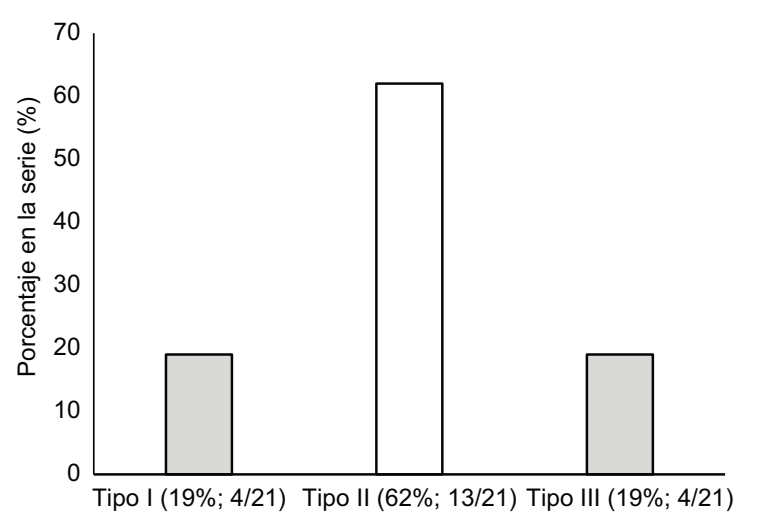

Figura 1. Distribución de las lesiones por disparo de escopeta, según la clasificación de Trunkey y Blaisdel/5 (Fuente: registro de la Sección de Cirugía de Trauma, Departamento de Cirugía General, Hospital Central Militar, SEDENA, Ciudad de México).

índice de gravedad anatómica. La mayor parte de nuestros pacientes presentaron LE de tipo II (62\%; 13/21), seguida por las lesiones de tipo I (19\%; 4/21), siendo las menos frecuentes las de tipo III (19\%; 4/21) (Fig. 1).

Debido a que todos los pacientes que se reciben en el departamento de urgencias de nuestra institución se manejan con los protocolos ATLS $^{\circledR}$ del American College of Surgeons, en el $100 \%$ de ellos (21/21) se colocaron líneas intravenosas periféricas. En 14 pacientes (32\%) se insertaron una o dos sondas pleurales, mientras que en cinco se colocó un catéter venoso central $(24 \%)$ y cuatro (19\%) fueron intubados orotraquealmente. Es notable que en un paciente $(5 \%)$ con trauma facial complejo se realizó una cricotiroidotomía por punción con técnica de Seldinger. Se realizó Untrasound Focused Assessment with Spund in Trauma (USG-FAST) en nueve pacientes (43\%), 20 se sometieron a valoración radiológica simple portátil (95\%) y en todos los pacientes de la serie (100\%; $21 / 21)$ se realizó tomografía computada simple o contrastada.

Aunque todos los pacientes fueron sujetos a intervención quirúrgica en nuestra institución en algún momento de su estancia hospitalaria, cinco $(24 \%)$ ya habían sido operados en los hospitales de referencia (cuatro por laparotomía exploradora y uno por toracotomía posterolateral izquierda). En dos pacientes (10\%) se realizó exploración cervical, siete (33\%) requirieron toracotomía y 17 (81\%) demandaron laparotomía exploradora primaria o de segunda revisión para su abordaje terapéutico. Cinco pacientes requirieron traqueostomía, realizándose cuatro de ellas por vía percutánea asistida por broncoscopía en la unidad de cuidados intensivos y una en el quirófano. Un paciente $(5 \%)$ requirió reconstrucción facial compleja secuencial con fijadores externos faciales y seis (29\%) requirieron procedimientos reconstructores con limpiezas quirúrgicas secuenciales (6/6), injertos cutáneos (4/6) y colgajos libres o pediculados (2/6). En 16 pacientes $(76 \%)$ se llevaron a cabo procedimientos secuenciales, con una media de 5.3 (rango: 2-17) procedimientos por paciente, la mayoría de ellos en tejidos blandos (9/16) y el abdomen (8/16). Como procedimientos adyuvantes no quirúrgicos se realizaron dos arteriografìas con angioembolización selectiva (10\%) y cuatro drenajes percutáneos de colecciones cavitarias (19\%).

Se computaron 100 lesiones a órganos específicos, con una media de 4.8 lesiones por paciente. Los órganos más comúnmente lesionados fueron la piel y las faneras (100\%; $21 / 21)$, seguidas por los traumatismos al órgano vascular periférico, hígado, pulmón (7/21; 33\% cada uno de ellos), yeyuno-íleon y colon (5/21; $24 \%$ cada uno). La distribución de la totalidad de las lesiones específicas se muestra en la tabla 1.

La estancia hospitalaria media fue de $37.1 \pm 28.7$ días (rango: 3-109). Un paciente con trauma facial complejo permaneció encamado 3 días y sucumbió por sepsis y falla orgánica múltiple. La estancia media en la unidad de cuidados intensivos fue de $20.3 \pm 22.5$ días (rango: 3-99) y la duración media de la asistencia ventilatoria mecánica fue de $16.1 \pm 20.8$ días

La tasa global de morbilidad fue significativamente alta y se ubicó en el orden del $82 \%$ (17/21). Se computaron 106 complicaciones específicas, con una media de cinco complicaciones por paciente. Las complicaciones específicas más frecuentes fueron de carácter infeccioso, figurando entre ellas la infección superficial o profunda del sitio quirúrgico (76\%; 16/21), la infección de vías urinarias (71\%; $15 / 21)$, la neumonía asociada al ventilador y la sepsis (43\%; 9/21 cada una). La falla orgánica que se presentó con más consistencia fue la insuficiencia renal $(38 \% ; 8 / 21)$, seguida por la falla orgánica múltiple (33\%; $7 / 21)$ y la falla respiratoria aguda (24\%; $5 / 21)$. En notable hacer mención de que en dos casos $(10 \%)$ hubo embolia de proyectil de posta pequeña (birdshots), una hacia la circulación esplácnica gastropancreática y otra hacia la circulación distal del miembro pélvico derecho, ambas curiosamente sin repercusión isquémica. La distribución de la totalidad de las complicaciones específicas se muestra en la tabla 2.

La tasa global de mortalidad fue del $24 \%$ (5/21). Las causas de muerte fueron falla orgánica múltiple en tres pacientes (14\%), sangrado no coagulopático en 
Tabla 1. Lesiones a órganos específicos en los pacientes con lesiones por disparo de escopeta

\begin{tabular}{|c|c|}
\hline Órgano específico lesionado & n/N (\%) \\
\hline Piel y faneras & $21 / 21(100)$ \\
\hline Órgano vascular periférico & 7/21 (33) \\
\hline Hígado & 7/21 (33) \\
\hline Pulmón & 7/21 (33) \\
\hline Parrilla costal & 6/21 (29) \\
\hline Yeyuno-íleon & $5 / 21(24)$ \\
\hline Colon & $5 / 21(24)$ \\
\hline $\begin{array}{l}\text { Órgano vascular torácico (otro diferente } \\
\text { a aorta y vena cava) }\end{array}$ & $4 / 21(19)$ \\
\hline Órgano vascular abdominal & $4 / 21(19)$ \\
\hline Bazo & 4/21 (19) \\
\hline Pelvis ósea & $3 / 21(14)$ \\
\hline Columna vertebral y médula espinal & $3 / 21(14)$ \\
\hline Esófago (cervical o torácico) & $3 / 21(14)$ \\
\hline Riñón & $3 / 21(14)$ \\
\hline Estómago & 2/21 (10) \\
\hline Recto & 2/21 (10) \\
\hline Órgano vascular cervical & 2/21 (10) \\
\hline Páncreas & 2/21 (10) \\
\hline Corazón y pericardio & 2/21 (10) \\
\hline Genitales externos & 2/21 (10) \\
\hline Laringe y tráquea & $2 / 21(10)$ \\
\hline Uréter & $1 / 21(5)$ \\
\hline Bronquios & $1 / 21(5)$ \\
\hline Esqueleto facial & $1 / 21(5)$ \\
\hline Hipofaringe & $1 / 21(5)$ \\
\hline
\end{tabular}

Total: 100 lesiones específicas (4.8 lesiones por paciente)

Fuente: registro de la Sección de Cirugía de Trauma, Departamento de Cirugía General, Hospital Central Militar, SEDENA, Ciudad de México.

un paciente $(5 \%)$ y falla respiratoria refractaria a las estrategias ventilatorias en un paciente (5\%). El sitio más común de fallecimiento fue la unidad de cuidados intensivos $(3 / 21 ; 14 \%)$, mientras que dos pacientes sucumbieron en la unidad de hospitalización regular. Es notable comentar que los cuatro pacientes con LE tipo III fallecieron, con una mortalidad específica del $100 \%$ para este tipo de lesiones, mientras que un paciente con LE tipo II falleció, con una mortalidad específica del $7 \%$ (1/13). No hubo decesos en el grupo de pacientes con LE tipo I.
Tabla 2. Tasa global de morbilidad y morbilidad específica en pacientes con lesiones por disparo de escopeta

\begin{tabular}{|c|c|}
\hline Complicación específica & $\mathrm{n} / \mathrm{N}(\%)$ \\
\hline Infección del sitio quirúrgico & $16 / 21(76)$ \\
\hline Infección urinaria & $15 / 21(71)$ \\
\hline Neumonía asociada al uso de ventilación mecánica & $9 / 21(43)$ \\
\hline Sepsis & $9 / 21(43)$ \\
\hline Insuficiencia renal & $8 / 21(38)$ \\
\hline Falla orgánica múltiple & $7 / 21(33)$ \\
\hline $\begin{array}{l}\text { Síndrome de insuficiencia } \\
\text { respiratoria progresiva del adulto }\end{array}$ & $5 / 21(24)$ \\
\hline Sangrado (causa técnica, no coagulopática) & $5 / 21(24)$ \\
\hline Empiema & $4 / 21(19)$ \\
\hline Coagulopatía & $4 / 21(19)$ \\
\hline Eventración & $3 / 21(14)$ \\
\hline Obstrucción intestinal & $3 / 21(14)$ \\
\hline Síndrome depresivo mayor & $3 / 21(14)$ \\
\hline Neumatocele & $2 / 21(10)$ \\
\hline Fistulización intestinal & $2 / 21(10)$ \\
\hline Miofascitis necrotizante & $2 / 21(10)$ \\
\hline Absceso pulmonar & $2 / 21(10)$ \\
\hline Embolia de misil & $2 / 21(10)$ \\
\hline Pseudoaneurisma de órgano vascular periférico & $2 / 21(10)$ \\
\hline Fístula pancreática & $1 / 21(5)$ \\
\hline Muerte cerebral & $1 / 21(5)$ \\
\hline Estenosis subglótica & $1 / 21(5)$ \\
\hline
\end{tabular}

Total: 106 complicaciones específicas (5 lesiones por paciente)

Tasa global de morbilidad: 82\% (17/21)

Fuente: registro de la Sección de Cirugía de Trauma, Departamento de Cirugía General,

Hospital Central Militar, SEDENA, Ciudad de México.

\section{Discusión}

Las LE son una epidemia infrecuente, pero ubicua y prevenible, que ocasionan no solo un grave daño mental y anatómico a la víctima, sino que también perjudican emocionalmente y financieramente a la sociedad. Esta infrecuencia ha generado inexperiencia en los centros de trauma urbanos y ha ocasionado que el cirujano no razone o desestime el mecanismo de lesión tan peculiar de las LE, lo que lleva a un abordaje inadecuado y a una falla para definir el cuadro general de lesiones ${ }^{1,4,6,7}$.

Es esencial que desde un primer momento el médico que atiende a un paciente con LE comprenda 
que el patrón lesional y la gravedad del traumatismo dependen críticamente de la distancia de disparo, del calibre del tubo de la escopeta y del tamaño de las postas $^{7,8}$. Así, se puede encontrar un amplio rango de heridas, que van desde lesiones puntiformes superficiales inducidas por postas pequeñas aisladas hasta lesiones destructivas profundas, con concentración de varios misiles pequeños o medianos en un área limitada o generadas por postas sólidas grandes ${ }^{5,7-9}$.

En términos generales, la comprensión que tiene el cirujano de la balística de las LE es muy pobre. Una escopeta es un tubo liso incluido en un sistema de sujeción y puntería, que posee una recámara, que aloja un cartucho conformado por una concha de plástico o cartón, un mecanismo de ignición en su cauda, un propelente explosivo y una carga de postas que convencionalmente pesan entre 5.71 y 45.5 gramos, las cuales, en función de su tamaño, se conocen como birdshots (pequeñas), buckshots (medianas) o slugs (sólidas grandes). Es preciso comprender también que cada posta puede generar una cavitación permanente (tracto de lesión o intrusión), que depende de la masa del misil, o causar una cavitación temporal inicial excéntrica y otra de naturaleza temporal tardía concéntrica, que se relaciona directamente con la velocidad del proyectil (la mayoría de las escopetas disparan misiles con velocidades que oscilan entre 556 y 402 m/s). La longitud del cañón, la dimensión de la apertura de su boca y la distancia de disparo definen en gran parte la concentración de las postas y, por consecuencia, la transmisión de la energía en el blanco. Se calcula que la energía transmitida por cada posta individual alcanza el orden de 880 a $3700 \mathrm{~J}$, lo cual explica el gran poder de destrucción cuando un gran número de ellas confluyen en un área definida, como en los disparos de corto rango. Adicionalmente, hay otros factores que definen el patrón lesional, como el perfil (forma y material del misil, fragmentación), fenómenos balísticos externos (precesión, nutación, tumbling y yaw), características anatómicas y físicas de los tejidos blanco, y elementos mitigantes o modificadores, como la ropa y la armadura corporal militar,3,5,6-8,10-12.

Aunque ha habido múltiples intentos para clasificar estas lesiones, ninguna ha sido capaz de normar la conducta ni establecer con certeza la necesidad de tratamiento quirúrgico. García-Núñez, et al., ${ }^{3}$ en una revisión de la literatura concerniente al tema, menciona que Asensio, del Grupo de Trauma de Los Ángeles, propuso en 2004 una modificación a la clasificación de Trunkey y Blaisdell ${ }^{5}$ basada en la distancia de disparo, haciendo una propuesta de abordaje sustentada en la experiencia clínica:
- Tipo I: llamadas también de «rango lejano». Son disparos a más de 7 yardas de distancia y por lo habitual se requieren cuidados superficiales de las heridas y extracción de las postas accesibles al operador. En general, el pronóstico es bueno para la vida y la función, y la mortalidad estimada es del 0-5\%.

- Tipo II: también denominadas de «rango intermedio». La distancia de disparo es de 3-7 yardas hacia la víctima. Las lesiones son profundas, se requiere un abordaje diagnóstico minucioso y la necesidad de intervención quirúrgica temprana es elevada. El pronóstico para la vida y la función es reservado, con una tasa de mortalidad del $5-10 \%$.

- Tipo III: conocidas también como de «rango corto». La distancia de disparo es menor de 3 yardas. Por lo general hay destrucción profunda de la anatomía. Todos los pacientes requieren intervención operatoria agresiva y muchos deben operarse bajo la política de la estrategia de control de daños. El pronóstico para la función es uniformemente malo y la tasa de mortalidad alcanza el $15-20 \%$. Este tipo de lesión es la causa de la muerte en el $85 \%$ de los casos de mortalidad por LE.

En nuestra serie, la mayoría de las víctimas de LE fueron varones jóvenes, lo cual es congruente con lo reportado en las grandes series epidemiológicas para este tipo de lesiones y el trauma en general $\left.\right|^{1,2,5,13}$. Debido a las actuales condiciones socioeconómicas y demográficas del país, donde es fácil obtener de forma clandestina armamento de alto poder, las LE representan una minoría de los traumatismos por arma de fuego, por lo cual nuestro hospital, a pesar de ser un centro de trauma militar y urbano que recibe casos de otros escalones militares nacionales, admite una media de 0.1 pacientes por mes, procedentes principalmente de la arena suburbana. Esta casuística es comparable a la reportada por Schellenberg, et al. ${ }^{8}$, quienes señalan para un centro de trauma de nivel I de los Estados Unidos una incidencia de 0.5 casos al mes. Esto puede llevar a desarrollar poca experiencia por los médicos de contacto y a instituir procedimientos inadecuados de abordaje diagnóstico y terapéutico, con potencial perjuicio de los índices pronósticos de sobrevida y funcionalidad.

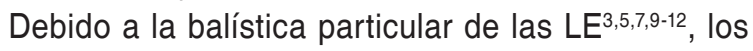
pacientes de nuestra serie arribaron en general estables desde el punto de vista fisiológico (RTS6.91 \pm 1.11 ), pero con lesiones de alto índice de gravedad 
anatómica (ISS $44.5 \pm 13.6)^{14}$. El mecanismo particular de la lesión también es causa del abordaje diagnóstico y terapéutico agresivo en el departamento de urgencias, donde en una tercera parte de los pacientes se tuvieron que emplear sondas pleurales y en una quinta parte fue requerida la intubación endotraqueal. El uso de tomografía computada fue necesario en el $100 \%$ de los casos, ya que por la clínica fue imposible definir la anatomopatología del daño, condición lógicamente impuesta por el impacto de múltiples postas. Sin embargo, este es el momento ideal para hacer una pausa de precaución, enfatizando que estos pacientes son muy sensibles a la mala interpretación de los hallazgos radiológicos - condición descrita en la literatura como VOMIT (Victim of Modern Imaging Technology)—, por lo cual aquí la colaboración entre el cirujano y el radiólogo es crítica ${ }^{15}$.

Como consecuencia esperada de la dispersión o confluencia de las postas en la víctima, afectando amplias regiones corporales o transmitiendo una gran magnitud de energía en un área anatómica reducida ${ }^{3-5,7,8,10,12}$, encontramos en nuestro estudio una enorme cantidad de lesiones a órganos específicos y un elevado promedio de órganos lesionados por paciente (4.8 lesiones por paciente), lo cual también demandó la realización de 49 procedimientos primarios (2.3 procedimientos por paciente) y que en el $76 \%$ se llevaran a cabo una media de 5.3 procedimientos quirúrgicos secuenciales. La necesidad de múltiples intervenciones operatorias ha sido descrita en diversos estudios de la literatura, debido a la amplitud del daño causado por la $L^{4,6}$ 8,10-12,16,17. Los factores antes señalados explican con claridad los índices pronósticos obtenidos. Se observaron unas prolongadas estancias hospitalaria (37.1 \pm 28.7 días) y en la unidad de cuidados intensivos (20.3 \pm 22.5 días), así como un número significativo de días durante los que los pacientes requirieron ventilación mecánica (16.1 \pm 20.8 días). La morbilidad se ubicó en el orden del 82\% (17/21), siendo las complicaciones específicas más frecuentes aquellas de carácter infeccioso. Consideramos que la grave disrupción anatómica, la prolongada estancia hospitalaria, la inmovilización y la desnutrición de los pacientes, el nivel de monitorización invasiva y la gran cantidad de procedimientos que se practicaron en las víctimas de $\mathrm{LE}^{15-17}$ hacen manifiesto el carácter del trauma como una enfermedad inmunodepresora, que convierte a los pacientes en un fácil blanco de infecciones relacionadas con el cuidado de la salud y que tiene una alta tasa de complicaciones no infecciosas y una significativa mortalidad, reflejo de la evolución tórpida que siguen muchos de estos pacientes.

La falla orgánica es otro problema que los cirujanos comprometidos con el cuidado del paciente superviviente deben encarar en alguna etapa de la estancia hospitalaria. En la serie, encontramos como falla orgánica más común la insuficiencia renal (38\%; $8 / 21)$, seguida por la falla multiorgánica (33\%; 7/21). Se sabe que la inflamación sistémica y la sepsis son inductoras y promotoras independientes de la falla renal aguda, debido a la producción de citocinas causantes de nefritis, lesión mitocondrial por peroxinitratos, constricción microvascular mediada por el sistema renina-angiotensina-aldosterona, aumento de la permeabilidad vascular por citotoxicidad humoral y celular, y estrés oxidativo del órgano mediado citológicamente. Por otro lado, estas mismas condiciones, aunadas al fenómeno de isquemia-reperfusión inducido por la reanimación del paciente traumatizado y a la rabdomiólisis consecutiva a la lesión extensa de tejidos blandos, pueden no solo conducir al paciente a la falla renal exclusiva, sino que también se pueden reflejar en insuficiencias orgánicas variadas, tal como fue en nuestro estudio $4,5,7,10,18,19$.

La tasa de mortalidad no fue despreciable en nuestra serie. Fallecieron cinco pacientes, de los cuales tres (60\% de los decesos) se debieron a falla orgánica múltiple. Un paciente presentó sangrado no coagulopático de origen retroperitoneal que cedió al empaquetamiento y embolización angiográfica selectiva, pero que llevó al paciente a un estado de choque refractario y a su muerte. Un último paciente falleció por insuficiencia respiratoria progresiva. Es especialmente importante mencionar que ningún paciente con LE de tipo III sobrevivió, con una tasa específica de mortalidad de $100 \%$ (4/4), lo cual no representa un dato sorprendente en el análisis de estas lesiones, pues la alta transmisión de energía, la grave disrupción anatómica, las múltiples intervenciones diagnósticas y terapéuticas, y los hits secuenciales, así como la monitorización invasiva y la respuesta proinflamatoria e inmunodepresora que acarrean como consecuencia en las víctimas de estos mecanismos de lesión, son factores conocidos en la literatura como condicionantes relacionados 0 predictores independientes de alta mortalidad ${ }^{1,2,4,5,7,8,10,12,18-21}$.

Se deben considerar las limitaciones de este estudio. Aunque se trata de un análisis de la casuística de un centro de trauma militar y urbano nacional, la frecuencia de las LE es baja y esto limitó el universo 
de estudio. Además, la distancia de disparo no pudo ser definida con precisión, ya que esta información se obtuvo del interrogatorio directo de la víctima, con toda la imprecisión que esto conlleva. Finalmente, el carácter retrospectivo y unicéntrico del estudio acarrea limitaciones inherentes al diseño. En un futuro, estas limitaciones pueden mitigarse con un estudio multicéntrico y prospectivo, que clarifique los datos nacionales requeridos para validar las propuestas y las conclusiones obtenidas.

Para finalizar, este estudio pone a la vista de la comunidad quirúrgica mexicana un análisis contemporáneo de la epidemiología, el patrón lesional, la evaluación diagnóstica y el manejo quirúrgico de los pacientes con LE admitidos al Hospital Central Militar, y representa en lo mejor de nuestro conocimiento, si no el único, el más grande estudio vertido a la literatura nacional en cirugía de trauma. Los pacientes que sufren LE son epidemiológicamente similares a aquellos que sufren traumatismos por proyectiles de otro tipo de armas de fuego y pueden ser abordados inicialmente de la misma manera, cubriendo las necesidades comunes. Sin embargo, el cirujano debe estar alerta a las limitaciones impuestas por la evaluación clínica y los estudios diagnósticos con los que se cuenta en la actualidad. Incluso la tomografía computada ha demostrado su alta tasa de fallas (falsos positivos y negativos) debido a los artefactos ocasionados por el conglomerado de postas ${ }^{15,16}$. Por lo anterior, recomendamos mantener un alto índice de sospecha de lesiones multiorgánicas y sujetar a los pacientes a periodos formales de observación hospitalaria pertinentes antes de egresarlos, incluso teniendo un resultado tomográfico aparentemente negativo para una lesión orgánica.

\section{Conclusiones}

Las LE son una condición traumática poco común, pero con una balística peculiar causante de un daño orgánico muy característico. Debido a su baja frecuencia, los cirujanos desarrollan poca experiencia en el manejo de estos casos, cometiendo errores en al abordar lesiones de alto grado de complejidad, lo cual afecta los índices pronósticos. Por la confluencia de varios factores mórbidos, con el mecanismo de lesión, la significativa disrupción anatómica, el alto número de lesiones específicas que presentan las víctimas, el gran volumen de intervenciones diagnósticas y terapéuticas a las cuales se sujetan, y el compromiso fisiológico que presentan durante su evolución, estos pacientes habitualmente incurren en prolongadas estancias hospitalarias y en la unidad de cuidados intensivos, necesidad de varios días de asistencia ventilatoria mecánica, gran tasa de morbilidad y alta frecuencia de complicaciones infecciosa, así como significativa mortalidad, condicionada principalmente por falla orgánica múltiple. Esto conlleva un gran consumo de recursos humanos, materiales y administrativos, por lo cual la intervención social con enérgicas estrategias de prevención primaria parece ser la conducta más conveniente para las instituciones que se encuentran comprometidas con la asistencia médico-quirúrgica de estos pacientes.

\section{Conflicto de intereses}

Los autores declaran que no hay relación financiera ni personal con ninguna persona u organización que pudiera generar conflicto de intereses.

\section{Responsabilidades éticas}

Protección de personas y animales. Los autores declaran que para esta investigación no se han realizado experimentos en seres humanos ni en animales.

Confidencialidad de los datos. Los autores declaran que han seguido los protocolos de su centro de trabajo sobre la publicación de datos de pacientes.

Derecho a la privacidad y consentimiento informado. Los autores han obtenido el consentimiento informado de los pacientes y/o sujetos referidos en el artículo. Este documento obra en poder del autor de correspondencia.

\section{Bibliografía}

1. Arcaute-Velázquez FF, García-Núñez LM, Noyola-Villalobos HF, Espinoza-Mercado F, Rodríguez-Vega CE. Mecanismos de lesión en actos de violencia extrema. Cir Cir. 2016;84:257-62.

2. Naghavi M, Marczak LB, Kutz M, Shackelford KA, Arora M, Global Burden of Disease 2016 Collaborators. Global mortality from firearms, 19902016. JAMA. 2018;320:1288.

3. García-Núñez LM. Cinemática del trauma. En: García-Núñez LM, editor. Cirugía avanzada en trauma. Principios teóricos y de aplicación práctica. Ciudad de México, México: Graphimedic; 2016. p. 8-13.

4. Dozier KC, Miranda MA, Kwan RO, Cureton EL, Sadjadi J, Victorino GP. Despite the increasing use of nonoperative management of firearm trauma, shotgun injuries still require aggressive operative management. J Surg Res. 2009;156:173-6.

5. Trunkey DD, Blaisdell FW. Trauma rounds: shotgun ballistics and shotgun injuries. Western J Med. 1978;129:149-55.

6. García-Núñez LM, Hernández-García EF. Manejo de lesiones generadas en trauma urbano: experiencia militar aplicada hacia casos específicos procedentes de la arena urbana. Cir Gen. 2012;34(Supl 1):59-61.

7. Hafertepen SC, Davis JW, Townsend RN, Sue LP, Kaups KL, Cagle KM. Myths and misinformation about gunshot wounds may adversely affect proper treatment. World J Surg. 2015;39:1840-7.

8. Schellenberg M, Inaba K, Heindel P, Forestiere MJ, Clark D, Matsushima K, et al. The diagnostic dilemma of shotgun injuries. Eur J Trauma Emerg Surg. 2019 Jun 10. doi: 10.1007/s00068-019-01168-4. [Epub ahead of print] 
9. Magaña-Sánchez IJ, Torres-Salazar JJ, García-Núñez LM, Núñez-Cantú O. Conceptos básicos de balística para el cirujano general y su aplicación en la evaluación del trauma abdominal. Cir Gen. 2011;33:48-53.

10. Cail K, Klatt $E$. The effect of intermediate clothing targets on shotgun ballistics. Am J Forensic Med Pathol. 2013;34:348-51.

11. Mansour DA, Elshaer AM, Elshazly MA. A new tailored protocol based on laparoscopy in the management of abdominal shotgun injuries: a case series study. Eur J Trauma Emerg Surg. 2018 Sep 24. doi: 10.1007/ s00068-018-1015-7. [Epub ahead of print]

12. Rhee PM, Moore EE, Joseph B, Tang A, Pandit B, Vercruysse G. Gunshot wounds: a review of ballistics, bullets, weapons, and myths. J Trauma Acute Care Surg. 2016;80:853-67.

13. Demetriades D, Murray J, Sinz B, Myles D, Chan L, Sathyaragiswaran L, et al. Epidemiology of major trauma and trauma deaths in Los Angeles County. J Am Coll Surg. 1998;87:373-83.

14. Palmer CS, Gabbe BJ, Cameron PA. Defining major trauma using the 2008 Abbreviated Injury Scale. Injury. 2015;47:109-15.

15. García-Núñez LM, González L, Cabello-Pasini R, Lever-Rosas CD, Rosales-Montes E, Padilla-Solís R. Síndrome del Victim of Modern Imaging Technology (VOMIT). An Radiol Mex. 2006;1:3-7.
16. Asensio JA, Petrone P, García-Núñez LM, Kimbrell B, Kuncir E. Multidisciplinary approach for the management of complex hepatic injuries AAST-OIS grades IV-V: a prospective study. Scand J Surg. 2007; 96:214-20.

17. García-Núñez LM, González L, Cabello-Pasini R, Magaña-Sánchez IJ, Pérez-Aguirre J. Grapado quirúrgico en la cirugía de control de daños del paciente gravemente traumatizado. Rev Biomed. 2006; 17:124-31.

18. Don Bosco D, Gangalal GM, Rao S, Chakrapani AT. Acute kidney injury in severe trauma patients; a record-based retrospective study. Adv J Emerg Med. 2019;3:22.

19. Hutchings SD, Naumann DN, Hopkins P, Mellis C, Riozzi P, Sartini S, et al. Microcirculatory impairment is associated with multiple organ dysfunction following traumatic hemorrhagic shock: the MICROSHOCK Study. Crit Care Med. 2018;49:889-6.

20. Croce M. AAST statement of fire injury - one surgeon's perspective. J Trauma Acute Care Surg. 2018;85:427-8.

21. Maa J, Darzi A. Firearm injuries and violence prevention - the potential power of a general surgeon's report. New Engl J Med. 2018;379:408-10. 